\title{
Alignment Issues in Photonic Crystal Device Fabrication
}

\author{
René M. de Ridder, Cazimir G. Bostan, Frank J. van Soest, and Vishwas J. Gadgil \\ University of Twente, MESA ${ }^{+}$Research Institute, Integrated Optical MicroSystems (IOMS) group, \\ * University of Twente, MESA ${ }^{+}$Research Institute, Faculty of Science and Technology, \\ P.O.Box 217,7500 AE Enschede, the Netherlands,e-mail:R.M.deRidder@utwente.nl
}

\begin{abstract}
An important requirement in the fabrication of photonic crystal structures is the correct relative alignment of structural elements. Accuracy should be in the order of some tens of nanometres. Some of the options for providing such accuracy will be discussed. Examples will be given of aligning defects with respect to a predefined $2 \mathrm{D}$ lattice, aligning access waveguides with respect to a small local photonic crystal structure, and the alignment of successive periodically structured layers in a 3D "woodpile" structure.
\end{abstract}

Keywords: photonic crystal slab, woodpile structure, alignment errors, laser interference lithography, focused ion beam.

\section{INTRODUCTION}

Several technologies are used for fabricating photonic crystal $(\mathrm{PhC})$ structures. They can be divided into two main classes: bottom-up (self-organisation, e.g. artificial inverted opals, e.g. [1]) and top-down (definition of designed patterns). Well-defined defects are needed in most cases for obtaining a desired functionality. Topdown fabrication approaches are best suited for creating functional defects. Some important fabrication technologies are based on direct e-beam lithography e.g. [2] or, more recently, deep-UV lithography [3], which allow definition of defects simultaneously with the $\mathrm{PhC}$ lattice. Although very good results have been obtained using these lithographic methods, they are not always convenient because they require extensive calibration due to electronic or optical proximity effects. Direct e-beam writing has the better resolution, but it is not very suitable for defining extended structures since the sequential writing process is relatively slow. Although $\mathrm{PhC}$ structures can, in principle, provide a large functionality on a rather small area of a few 100 or $1000 \mu \mathrm{m}^{2}$, for practical reasons the optical chips need to be several $\mathrm{mm}^{2}$, so that additional optical waveguides of several $\mathrm{mm}$ length must be provided on chip for accessing the PhC structures. Problems with proximity effects can be avoided by defining the regular $\mathrm{PhC}$ lattice and the defects and the access waveguides using separate processes. However, this introduces the problem of aligning these non-periodic features with respect to the lattice. A similar alignment issue arises in fabricating three-dimensional $\mathrm{PhC}^{\prime} \mathrm{s}$ in a layer-by-layer fashion, where successive layers must be mutually aligned.

In this paper we will discuss some fabrication techniques, based on focused ion beam (FIB) processing, laser interference lithography (LIL) and conventional (near-UV) mask-based lithography, that may provide a fast and relatively straightforward pattern definition without the need for proximity correction. The basic idea is to combine the strong points of each of the component technologies: FIB for defining the smallest features on a tiny area, LIL for defining extremely regular periodic structures over a large area, and conventional mask lithography for defining larger, generally non-periodic, structures like wide access waveguides, possible membrane supports, etc.

In section 2 we will discuss two different approaches for fabricating quasi two-dimensional PhC structures (PhC slabs), viz. (1) LIL definition of an extended PhC lattice in a metal hard mask, where defects are defined by local FIB-induced platinum deposition, and (2) access waveguide definition with conventional mask lithography, combined with FIB etching of a small PhC structure in a metal hard mask. In section 3, a method will be shown for aligning periodic structures in different layers which form a 3D $\mathrm{PhC}$ of the so-called woodpile type.

\section{PHOTONIC CRYSTAL SLAB DEVICES}

A photonic crystal slab consists of a thin layer of high refractive index material which is perforated by a twodimensional lattice of holes having lower refractive index. These structures, first proposed by Meade et al. [4], and experimentally investigated by several authors, e.g. [5], can be designed to have a photonic stop band in the plane of the slab. Waveguides and resonators can be formed in these slabs by introducing deviations from the regular photonic crystal lattice -so-called defects-, which often consist of omitted (rows of) holes and/or holes having a different radius than the regular part of the lattice.

Earlier, we have reported the use of laser interference lithography (LIL) for defining a large area regular lattice of holes in a thin $\mathrm{Si}_{3} \mathrm{~N}_{4}$ membrane, and using local metal deposition using a focused ion beam (FIB) for

Part of this work was supported by the Dutch technology foundation STW. 


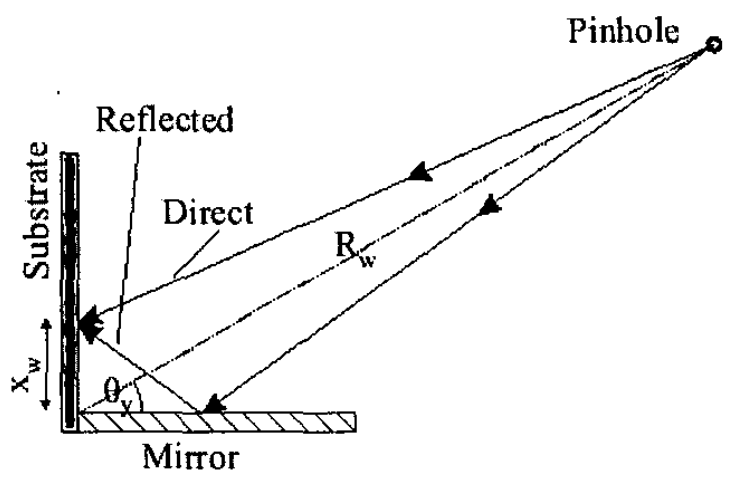

Figure 1. Laser Interference Lithography (LIL) using a Lloyd's mirror set-up.

defining defects consisting of missing rows of holes [6-8]. We use a very simple and stable LIL set-up using a Lloyd's mirror configuration as shown in Fig. 1. Using a $\lambda=266 \mathrm{~nm}$ UV laser light source, linear gratings can be produced with periods $\Lambda$ ranging from $142 \mathrm{~nm}$ to $765 \mathrm{~nm}$ by changing the angle of incidence $\theta$ of the two interfering beams by rotating the substrate-mirror assembly $(\Lambda \cong \lambda(2 \sin \theta))$. Two-dimensional patterns of photoresist pillars (rectangular or triangular lattice) are defined by a second exposure after rotating the substrate over $90^{\circ}$ or $60^{\circ}$. After depositing a thin metal film on top of this resist pattern and performing a lift-off process, a metal layer with an array of holes remains. Defects can be introduced by selectively filling some of the holes. Depending on the air filling factor of the $\mathrm{PhC}$, the defect definition must be accurate to a few tens of nm in order to avoid inadvertently partially filled holes. Using an imaging mode of the FIB machine, locations where defects should be produced can be determined with an accuracy down to $10 \mathrm{~nm}$. Then, using the FIB gas-assisted local metal deposition mode, holes can be filled selectively, with a resolution of 20 to $30 \mathrm{~nm}$. Such a locally modified metal pattern, which is used as a hard mask for etching the dielectric layer underneath, is shown in Fig. 2. Figure 3 shows a detail of the resulting structure, a thin silicon nitride photonic crystal slab with a line defect.

Although the LIL method works well to produce large uniform lattices, it has a drawback that the double exposure method for a triangular lattice will produce elliptical holes, which is undesirable for obtaining a wide stop band. This can be solved in principle by using a three beam interference exposure [9].

We have chosen to use FIB also for defining the regular lattice of holes in silicon on insulator substrates. Although the FIB process is relatively slow due to its sequential writing nature, this is not a very important issue in prototyping small $\mathrm{PhC}$ structures, especially in high refractive index contrast systems where the penetration depth of the electromagnetic field in the stop band is only a few rows of holes. Also, $\mathrm{PhC}$ waveguides still have relatively high propagation loss compared to photonic wires, e.g. $[3,10]$, leading to a strategy of fabricating small $\mathrm{PhC}$ cells interconnected by photonic wires or other waveguiding structures.

Due to its high resolution, the FIB process has the important advantage over LIL (and deep UV lithography) that arbitrary hole shapes having features in the order of $10 \mathrm{~nm}$ can be produced. In our modelling work we have found that using hexagonal holes may have advantages for designing low-loss PhC waveguides [11].

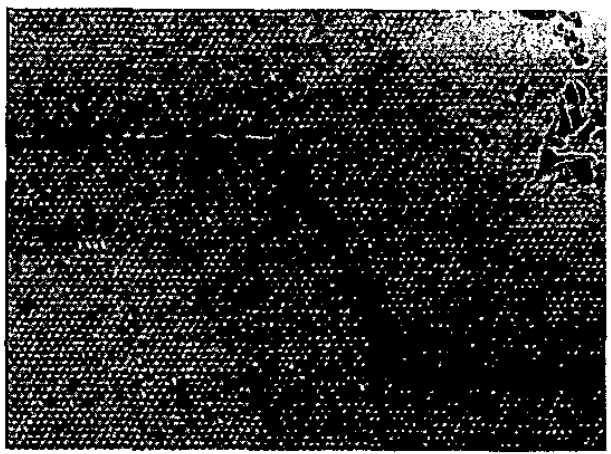

Figure 2. Scanning FIB image of triangular hole pattern $(a=560 \mathrm{~nm})$ defined by LIL in Cr mask, with line defect made by FIB-assisted Pt deposition filling selected holes.

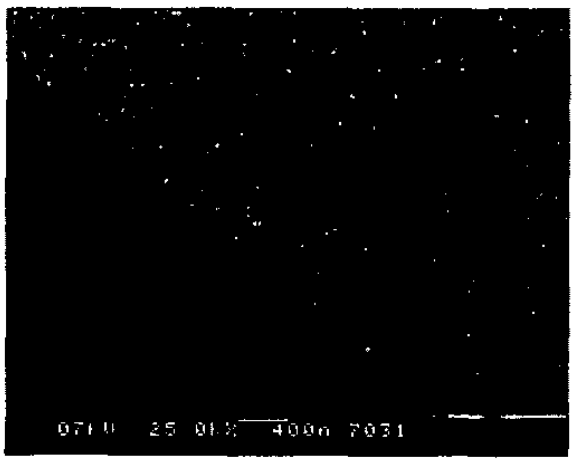

Figure 3.SEM image of silicon nitride photonic crystal slab with line defect. 

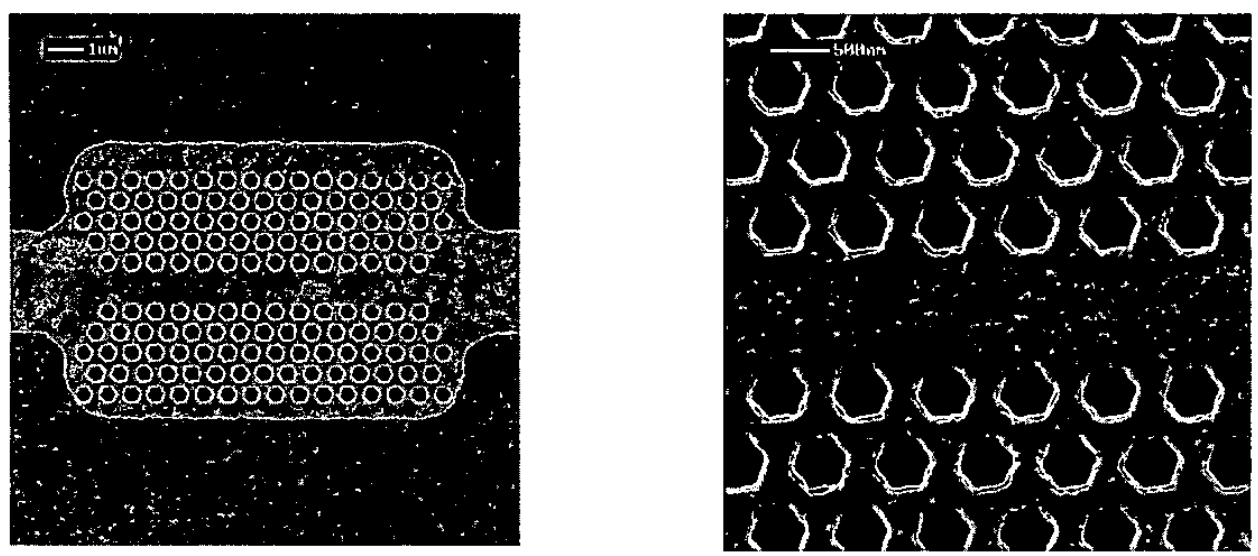

Figure 4. Left: FIB-etched hexagon-type photonic crystal pattern, aligned with waveguide pattern defined by conventional lithography in chromium. Right: detailed view.

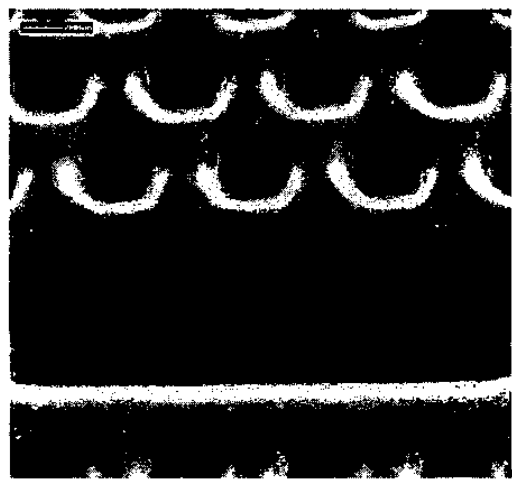

Figure 5. FIB cross-section showing the profile of top Si layer after RIE.

The photonic chips need to be much larger than the elementary $\mathrm{PhC}$ structures in order to be able to handle them in a simple way for optical characterisation. As it would be inconveniently time-consuming to define the resulting long access waveguides using FIB, we chose to define these access waveguides in a metal masking layer using conventional near UV mask lithography. Again exploiting the imaging feature and the high positioning accuracy of FIB, the PhC structure is easily aligned with the predefined waveguides as shown in Fig. 4. Performing FIB etching on a highly conductive metal layer prevents the occurrence of any localised charge accumulation and hence proximity effects. The $\mathrm{PhC}$ structure and the access waveguides are produced simultaneously in the silicon layer underneath the metal by a reactive ion etching process using an $\mathrm{SF}_{6} / \mathrm{CHF}_{3} / \mathrm{O}_{2}$ gas mixture at a pressure of $100 \mathrm{mTorr}$, tuned for high anisotropy and low roughness. The result is shown in Fig. 5 , where the cross-section has been revealed by etching a slit directly into the silicon with FIB.

\section{3D WOODPILE STRUCTURE}

Three-dimensional photonic crystals are interesting because they may have a full photonic bandgap. However, they are more difficult to fabricate than photonic crystal slabs. One of the most interesting 3D PhC structures is the so-called woodpile configuration, proposed by Ho et al. [12], illustrated in Fig. 6. It is probably the structure that is best suited for layer-by-layer fabrication since it can be built from layers that each have the shape of a simple linear grating, allowing a relatively straightforward defect definition [13]. Using silicon beams in air, a large full photonic bandgap up to $18 \%$ of the centre gap frequency may be obtained. This kind of structure has been fabricated for optical frequencies by multiple wafer bonding [13] and by using a true layer-by-layer method, e.g. [14]. It has been shown, both theoretically [15, 16] and experimentally $[17,18]$, that the woodpile structure is robust against structural variations.

Lateral misalignment of $10 \%$ of the period $\Lambda$ of the beams in a layer may decrease the bandgap by $30 \%$. The structure is quite tolerant for angular misalignment up to $5^{\circ}$ provided that all nominally parallel beams are rotated by the same amount. 


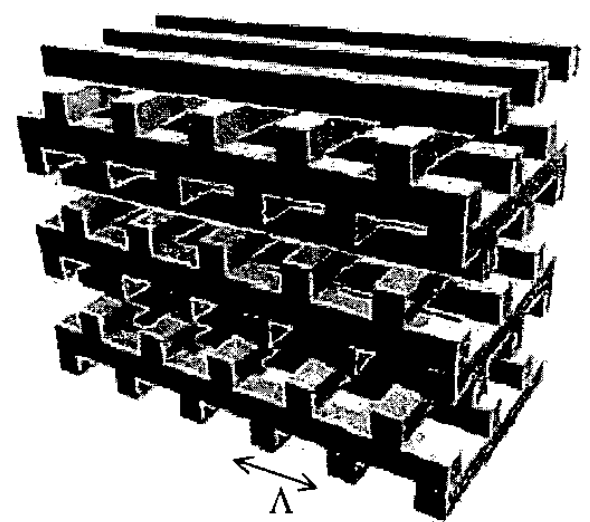

Figure 6. The woodpile structure.

However, it is this parallelism that may be compromised in a layer-by-layer fabrication method since each layer must be individually aligned with respect to the previously fabricated ones. If a relatively large photonic crystal is desired, requiring e.g. beams of length $L$, then a small angular misalignment $\theta$ may translate into a large, position dependent lateral misalignment up to $L \sin \theta$. For example in the case that $L=1 \mathrm{~mm}$ and $\Lambda=650 \mathrm{~nm}$ (typical for a bandgap around $1.5 \mu \mathrm{m}$ in a silicon/air structure), the maximum allowed angular misalignment resulting in a maximum lateral error of $0.1 \mathrm{~L}$, would be around $\theta=0.0035^{\circ}$.

We investigated the possibility for fabricating woodpile structures by stacking silicon layers that are patterned using LIL and reactive ion etching [19]. For this purpose we needed to be able to align a new LIL pattern with respect to an existing reference pattern on the silicon wafer. As shown in Fig. 7, the LIL set-up has three rotational degrees of freedom, of which rotation $\theta_{y}$ about the $y$-axis (defining $\Lambda$ ) and $\theta_{z}$ about the $z$-axis (defining angular misalignment) are the most critical ones. We control these angular alignments by observing the interference pattern that is produced by different diffraction orders of the reference grating, as indicated in the inset of Fig. 7, where the dashed light path produces the zero-order and the solid path the order -1 diffracted beam. Figure 8 shows the interference patterns for several values of alignment errors $\Delta \theta_{y}$ and $\Delta \theta_{z}$, indicating that an accuracy of $0.001^{\circ}$ can be obtained. Thus, the LIL pattern definition is expected to be suitable for fabricating large-area woodpile structures with good uniformity.

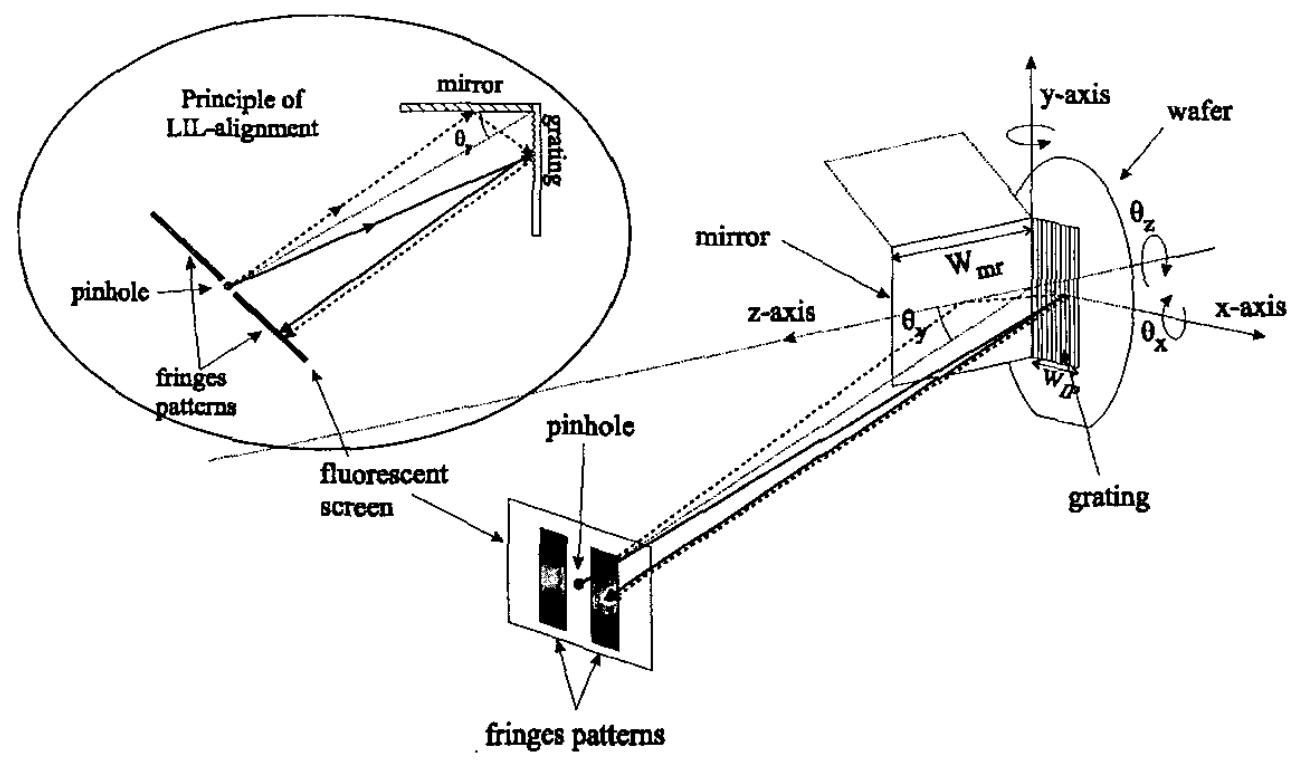

Figure 7. LIL alignment of new interference pattern with respect to an existing pattern. The main figure is a $3 D$ schematic showing the rotational degrees of freedom. Inset at upper left: principle of interference between $0^{\text {th }}$ and $-1^{\text {st }}$ order reflections from existing grating. 


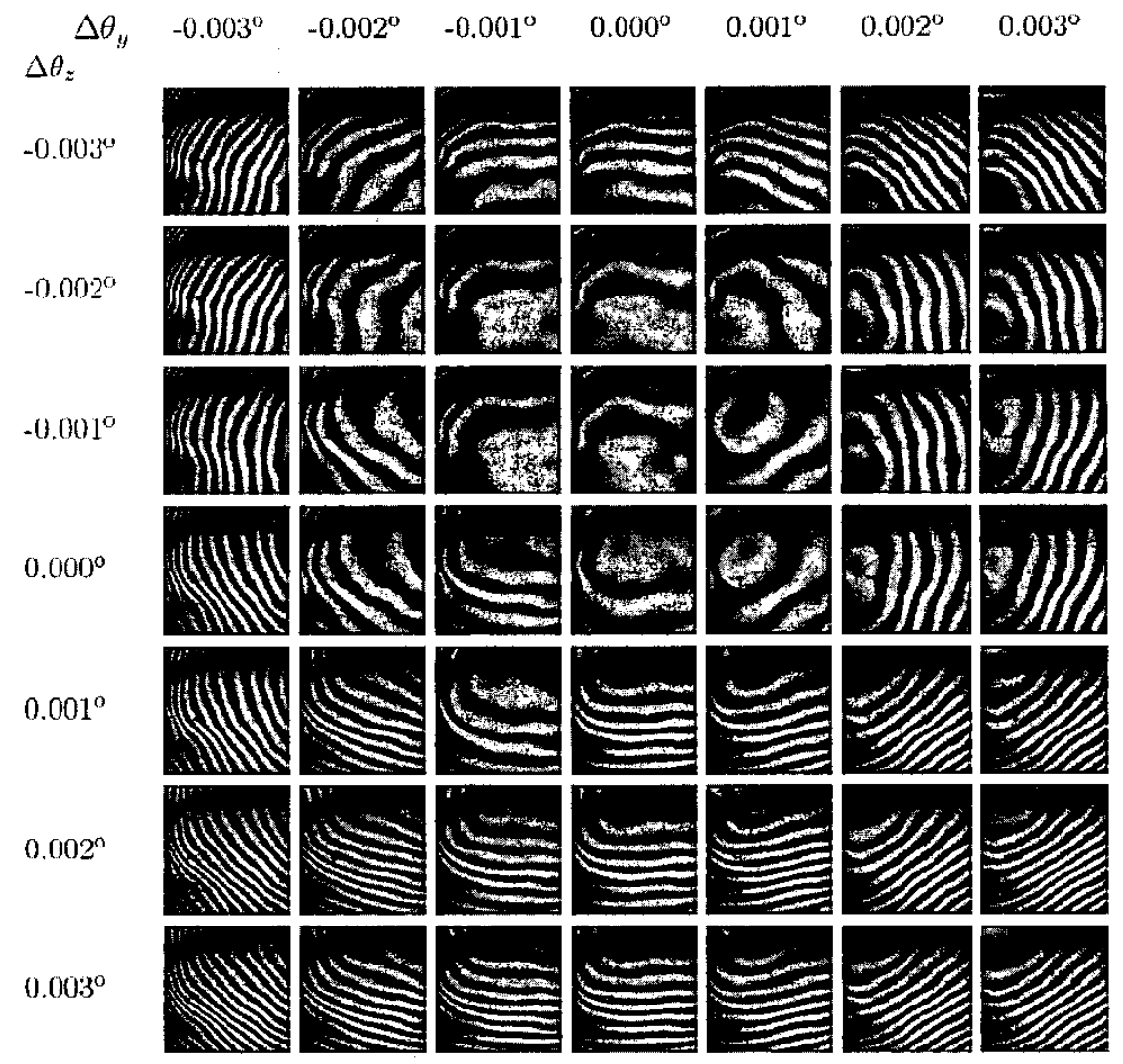

Figure 8. Different fringe patterns for several combinations of misalignment angles $\Delta \theta_{y}$ and $\Delta \theta_{z}$, as observed when a wafer with an existing grating is repositioned in the setup shown in Fig. 7.

\section{CONCLUSIONS}

We have argued that it can be advantageous to define large scale and nano-scale features of photonic crystal slab structures using separate optimized processes. Large-area uniform periodic structures can well be produced by laser interference lithography (LIL), and large non-periodic structures are conveniently defined using conventional near UV mask-based lithography. Focused ion beam (FIB) processing is in particular suited for defining nano-scale features. The resulting alignment requirements can be well fulfilled using FIB processing. Also, LIL can be used for producing periodic structures that are well aligned in period and in orientation with respect to a reference grating on a substrate. This makes it a promising technique for defining the periodic patterns in the individual layers of large 3D photonic crystals of the woodpile type.

\section{ACKNOWLEDGEMENTS}

The authors gratefully acknowledge the support of several colleagues in the fabrication of sample structures, in particular Henk van Wolferen for LIL processing and Henry Kelderman for general technology support.

\section{REFERENCES}

[1] J.E.G.J. Wijnhoven, W.L. Vos: Preparation of Photonic Crystals Made of Air Spheres in Titania, Science, vol. 281 , pp. 802-804, 1998.

[2] C.C. Cheng, A. Scherer: Fabrication of photonic band-gap crystals, J. Vac. Sci. Technol. B, vol. 13, pp. 2696-2700, 1995.

[3] W. Bogaerts, D. Taillaert, B. Luyssaert, P. Dumon, J. Van Campenhout, P. Bienstman, D. Van Thourhout, R. Baets, V. Wiaux, S. Beckx: Basic structures for photonic integrated circuits in Silicon-on-insulator, Optics Express, vol. 12, pp. 1583-1591, 2004. 
[4] R.D. Meade, A. Devenyi, J.D. Joannopoulos, O.L. Alerhand, D.A. Smith, K. Kash: Novel applications of photonic band gap materials: Low-loss bends and high Q cavities, J. Appl. Phys., vol. 75, pp. 4753-4755, 1994.

[5] T.F. Krauss, R.M. de la Rue: Optical characterization of waveguide based photonic microstructures, Appl. Phys. Lett., vol. 68, pp. 1613-1615, 1996.

[6] L. Vogelaar, W. Nijdam, H.A.G.M. van Wolferen, R.M. de Ridder, F.B. Segerink, E. Flück, L. Kuipers, N.F. van Hulst: Large area photonic crystal slabs for visible light with waveguiding defect structures: fabrication with focused ion beam assisted laser interference lithography, Advanced Materials, vol. 13, pp. 1551-1554, 2001.

[7] R.M. de Ridder, C.G. Bostan, H.A.G.M. van Wolferen, I. van Dorssen, L. Vogelaar, F.B. Segerink, L. Kuipers, N.F. van Hulst: Fabrication of photonic crystal slabs and defects using laser interference lithography and focused ion beam-assisted deposition, in Proc. ICTON 2002, Warsaw, Poland, pp. 14-19. April 2002.

[8] C.G. Bostan, R.M. de Ridder, I. van Dorssen, H.A.G.M. van Wolferen, L. Kuipers, N.F. van Hulst: Modeling and process design for laser interference lithography used in fabricating two-dimensional periodic structures, in Proc. ICTON 2002, Warsaw, Poland, p. 61, April 2002

[9] V. Berger, O. Gauthier-Lafaye, E. Costard: Photonic band gaps and holography, J. Appl. Phys, vol. 82, pp. 60-64, 1997.

[10] S.J. McNab, N. Moll, Y.A. Vlasov: Ultra low loss photonic integrated circuit with membrane-type photonic crystal waveguides, Optics Express, vol. 11, pp. 2927-2939, 2003.

[11] C.G. Bostan, R.M. de Ridder, V.J. Gadgil, H. Kelderman, L. Kuipers, A. Driessen: Design and fabrication of line-defect waveguides in hexagon-type SOI photonic crystal slabs, in Proc. Photonics Europe, Strasbourg, France, April 2004, SPIE. vol. 5450, paper 5450-39.

[12] K.M. Ho, C.T. Chan, C.M. Soukoulis, R. Biswas, M. Sigalas: Photonic bandgaps Photonic band gaps in three dimensions: New layer-by-layer periodic structures, Solid State Commun., vol. 89, pp. 413-416, 1994.

[13] S. Noda: Three-dimensional photonic crystals operating at optical wavelength region, Physica B, vol. 279, pp. 142-149, 2000.

[14] S.Y. Lin, J.G. Fleming, D.L. Hetherington, B.K. Smith, R. Biswas, K.M. Ho, M. Sigalas, W. Zubrzyski, S.R. Kurtz, J. Bur: A three-dimensional photonic crystal operating at infrared wavelengths, Nature, vol. 394, pp. 251-253, 1998.

[15] A. Chutinan, S. Noda: Effects of structural fluctuations on the photonic bandgap during fabrication of a photonic crystal, J. Opt. Soc. Am. B, vol. 16, 240-244, 1999.

[16] B. Gralak, M. de Dood, G. Tayeb, S. Enoch, D. Maystre: Theoretical study of photonic band gaps in woodpile crystals, Phys. Rev. E, vol. 67, 066601 (18), 2003.

[17] S. Ogawa, K. Tomoda, S. Noda: Effects of structural fluctuations on three-dimensional photonic crystals operating at near-infrared wavelengths, J. Appl. Phys., vol. 91, pp. 513-515, 2002.

[18] A. Feigel, B. Sfez: Overlapped woodpile photonic crystals, Appl. Optics, vol. 43, pp. 793-795, 2004.

[19] F.J. van Soest, H.A.G.M. van Wolferen, H.J.W.M. Hoekstra, R.M. de Ridder, K. Wörhoff, P.V. Lambeck: Laser interference lithography with highly accurate interferometric alignment, in Proc. $8^{\text {th }}$ Ann. Symp. IEEE/LEOS Benelux Chapter, Enschede, The Netherlands, pp. 269-272, November 2003. 